\title{
Critical exponents of EuTe from specific-heat and thermal-expansion measurements
}

\author{
E. Scheer ${ }^{a}$, J. Wosnitza ${ }^{a}$, H. v. Löhneysen ${ }^{a}$, R. Kürsch ${ }^{b}$, M. Lang ${ }^{b}$ and F. Steglich ${ }^{b}$ \\ "Physikalisches Institut, Universität Karlsruhe, W-7500 Karlsruhe, Germany \\ ${ }^{b}$ Institut für Festkörperphysik, Technische Hochschule Darmstadt, W-6100 Darmstadt, Germany
}

\begin{abstract}
The antiferromagnet EuTe shows a distinct cross-over behavior in the specific heat $C$ when approaching the Néel temperature $T_{\mathrm{N}}=9.8 \mathrm{~K}$. For $|t|=\left|\left(T-T_{\mathrm{N}}\right) / T_{\mathrm{N}}\right|>0.01$ the critical exponent of the specific heat, $C \alpha|t|^{-\alpha}$, is almost zero, $\alpha=0.0085 \pm 0.003$. Closer to $T_{\mathrm{N}}$, for $0.0015<|t|<0.01, C$ exhibits a different behavior with a surprisingly large positive $\alpha=0.38 \pm 0.1$, considerably exceeding even the $d=3$ Ising exponent $\alpha=0.11$. Measurements of the thermal expansion yield essentially the same cross-over behavior with $\alpha=0.002 \pm 0.01$ for $|t|>0.01$ and $\alpha=0.43 \pm 0.2$ for $0.001<|t|<0.03$. Although EuTe is considered to be a Heisenberg antiferromagnet, some anisotropy might be expected because of dipolar interactions, possibly causing the observed cross-over.
\end{abstract}

Of the Eu monochalcogenides, EuO and EuS are ferromagnets (fm) at low temperatures and correspond very well to the predictions for $3 \mathrm{~d}$-Heisenberg magnets in many static properties [1] while for dynamic properties dipolar interactions have to be taken into account [2]. EuSe shows complicated metamagnetic behavior [3]. EuTe orders antiferromagnetically (afm) with a Néel temperature $T_{\mathrm{N}}=9.8 \mathrm{~K}$ because the afm nextnearest neighbor interaction $J_{2} / k_{\mathrm{B}}=-0.16 \mathrm{~K}$ exceeds the $\mathrm{fm}$ nearest neighbor interaction $J_{1} / k_{\mathrm{B}}=0.06 \mathrm{~K}$ $[1,4]$. The magnetic structure is of the second kind (MnO structure) with the (111) planes as easy planes. The ratio between anisotropy field due to magnetic dipole-dipole interaction and interaction field is $H_{\mathrm{A}} / H_{\mathrm{E}} \approx 0.1$. In small magnetic fields $B_{\mathrm{f}} \approx 0.08 \mathrm{~T}$ (parallel [100]) EuTe flops into the canted phase with the spins still in the easy planes but directed nearly perpendicular to each other and to the applied magnetic field. Increasing field aligns the spins more and more and at the critical field, $B_{\mathrm{c}}=7.4 \mathrm{~T}$ at $T=0 \mathrm{a}$ second order phase transition to the paramagnetic state occurs. As a result of an additional in-plane anisotropy, the $[11 \overline{2}]$ directions are the easy directions $[5,6]$.

Here we report on high-resolution measurements of the specific heat and the thermal expansion of EuTe to investigate the critical behavior of an anisotropic antiferromagnet. For 3d-Heisenberg magnets with shortrange interaction, $\alpha=-0.12$ is obtained from renormalization-group (RG) calculations [7] and confirmed experimentally for EuS [8] and antiferromagnetic $\mathrm{RbMnF}_{3}[9]$.

The single crystals used in the experiment were obtained from K. Fischer at the Institut für Festkörperforschung, Forschungszentrum Jülich. They were grown in Ar atmosphere at $\sim 2200^{\circ} \mathrm{C}$ in closed tungsten crucibles. The specific-heat measurements were performed with a standard heat-pulse technique. The temperature resolution is better than $0.01 \mathrm{mK}$ at the afm transition.

The thermal expansion was measured with a capacitance dilatometer with sensitivity $\Delta L / L \approx 10^{-10}$. Length changes $\Delta L(T)=L(T)-L(1.4 \mathrm{~K})$ were detected in temperature intervals of about $30 \mathrm{mK} . \alpha_{1}(T)$ can be calculated directly via $\alpha_{1}(T)=\left[\Delta L\left(T_{2}\right) / L\left(T_{2}\right)\right.$ $-\left(\Delta L\left(T_{1}\right) / L\left(T_{1}\right)\right] /\left(T_{2}-T_{1}\right)$ with $T=\left(T_{1}+T_{2}\right) / 2$.

Fig. 1 shows the specific heat $C$ and linear thermal expansion coefficient $\alpha_{\mathrm{L}}$ of EuTe in the temperature range $8<T<12 \mathrm{~K}$. The data have been normalized to coincide at the transition temperature $T_{\mathrm{N}}=9.8 \mathrm{~K}$ and

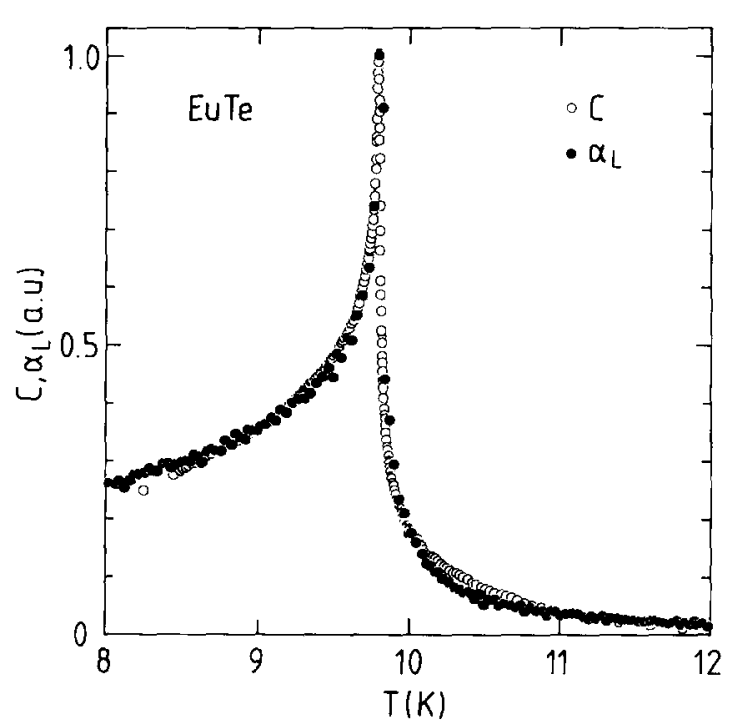

Fig. 1. Specific heat $C$ and linear thermal-expansion coefficient $\alpha_{\mathrm{L}}$ vs. temperature $T$ of EuTe for $8 \mathrm{~K}<T<12 \mathrm{~K}$. Both quantities have been normalized at $T_{\mathrm{N}}$ and at $12 \mathrm{~K}$. 


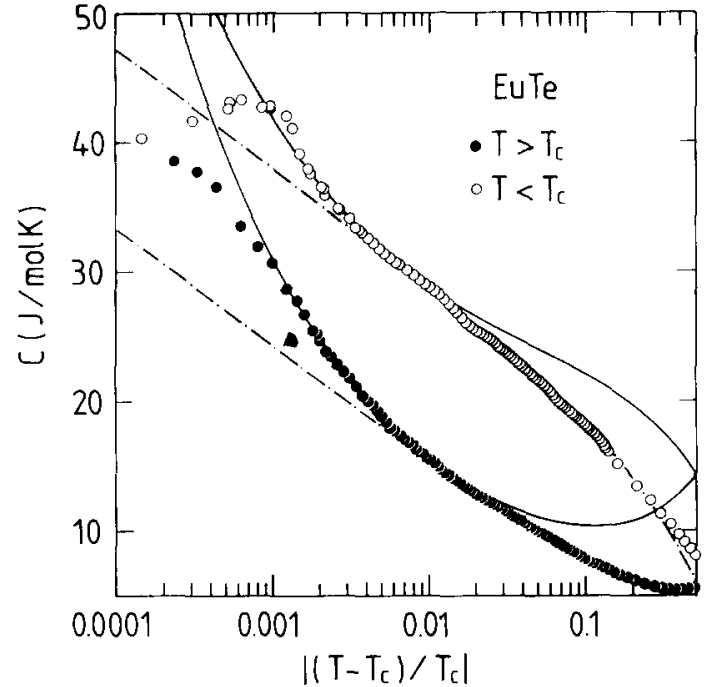

Fig. 2. Specific heat $C$ vs. the reduced temperature $|t|$ of EuTe. The lines are fits to eq. (1). Dashed-dotted line: $\alpha=0.0085$, solid line: $\alpha=0.38$.

at $12 \mathrm{~K}$, well outside the critical region. This visualizes the strong similarity of both quantities in the critical region.

The specific-heat data in the vicinity of $T_{\mathrm{N}}$ are evaluated with the function [8]

$C(t)=(A / \alpha)|t|^{-\alpha}+B+E t$

for $T>T_{\mathrm{c}}$ with $t=\left(T-T_{\mathrm{c}}\right) / T_{\mathrm{c}}, T_{\mathrm{c}}=T_{\mathrm{N}}$. The same function with the parameters $A^{\prime}, \alpha^{\prime}, B^{\prime}$, and $E^{\prime}$ is employed to describe the data for $T<T_{\mathrm{c}}$. RG calculations yield $\alpha=\alpha^{\prime}$ and $E=E^{\prime}$ [8]. The remaining parameters $\alpha, A, A^{\prime}, B, B^{\prime}$ and $E$ are obtained by fitting eq. (1) to both sets of data $t>0$ and $t<0$ to yield common best-fit parameters $\alpha$ and $E$, while $A, A^{\prime}$ and $B, B^{\prime}$ are allowed to differ from each other. $\alpha_{1}$ is evaluated with the same function.

Fig. 2 displays $C$ vs. $\log |t|$ for EuTe together with the fits. The clear increase of the slope of $C$ approaching $T_{c}$ for $0.001<|t|<0.01$ cannot be explained by sample inhomogeneitics which lead to a maximum for the data $t<0[10]$ but is an intrinsic feature of EuTe. This change of slope might indicate a cross-over to a different fixed point very close to $T_{c}$. While $\alpha=0.0085$ \pm 0.003 for $0.01<|t|<0.08$, one obtains $\alpha=0.38 \pm$ 0.1 for smaller reduced temperatures $0.0015<|t|<$ 0.01 (cf. fig. 2). Measurements performed on several samples confirm this. Evaluation of the thermal-expansion data yiclds $\alpha^{*}=0.002 \pm 0.01$ for $0.01<|t|<0.2$ and $\alpha^{*}=0.43 \pm 0.2$ for $0.001<|t|<0.03$. This crossover was not observed in previous work, although some deviations from Heisenberg behavior were found [3].
For the fit at smaller $|t|$, the constraint $B=B^{\prime}$ could not be fulfilled, therefore the apparent value of $\alpha$ only describes the increase of the critical exponent and is not a meaningful fit to the data. The large positive critical exponent of the specific heat is in contradiction to the expectation for isotropic magnets. Also, the magnetic-field dependence of $C$ and of the magnetic susceptibility show anisotropic behavior [11].

The deviations of $\alpha$ from Heisenberg behavior are thus attributed to the anisotropy arising from the magnetic dipole-dipole-interaction, which prevents the transversal spin fluctuations from growing to infinity. The complicated anisotropy in EuTe might be the reason that neither Ising $(\alpha=+0.11)$ nor $X Y$-behavior $(\alpha=-0.008)[7]$ is obscrved, but a transition with a large critiçal exponent $\approx 0.3-0.4$ very close to $T_{\text {. }}$. Another possible explanation for the observed crossover could be a transition of first order. From the thermal-expansion measurements, this possibility can neither be confirmed nor dismissed. Dynamic measurements should be employed to further elucidate the phase transition in EuTc.

As a final point, we mention that upon dilution of EuTe with $\mathrm{SrTe}$, the cross-over quickly vanishes. Already in $\mathrm{Eu}_{2} \mathrm{Sr}_{1}, \mathrm{Te}$ with $x=0.95$ a single exponent $\alpha$ is observed. $\alpha$ decreases towards -1 as $x$ approaches $x_{c}=0.5$, the eritical concentration for afm order [11].

We would like to thank K. Fischer for providing the high-quality single crystals necessary for this study.

\section{References}

[1] W. Zinn, J. Magn. Magn. Mater. 3 (1976) 23.

[2] J. Kötzler and W. Scheithe. J. Magn. Magn. Mater. $54-57$ (1986) 649.

[3] W.R. Johanson and D.C. McCollum, Phys. Rev. B22 (1980) 2435. K. Westerholt and G. Sobotta, J. Phys. F 13 (1983) 2371. K. Westerholt, J. Magn. Magn. Mater. 54-57 (1986) 721

[4] N.F. Oliveira, Jr., S. Foner. Y. Shapira and T.B. Reed, Phys. Rev. B 5 (1972) 2634.

[5] G. Will, S.J. Robert, H.A. Alperin and R. Nathans, J. Phys. Chem. Solids 24 (1963) 1679.

[6] J.W. Battles and G.E. Everett, Phys. Rev. B 1 (1970) 3021.

[7] J.C. Le Guillou and J. Zinn-Justin, Phys. Rev. Lett. 39 (1977) 95: Phys. Rev. B 21 (1980) 3976.

[8] A. Kornblit, G. Ahlers and E. Buehler, Phys. Rev. B 17 (1978) 282. A. Kornblit and G. Ahlers. Phys. Rev. B 11 (1975) 2678 .

[9] A. Kornblit and G. Ahlers, Phys. Rev. B 8 (1973) 5163.

[10] L.W. Kreps and S.A. Friedberg, J. Low Temp. Phys. 26 (1977) 317.

[11] E. Scheer, J. Wosnitza and H. v. Löhneysen, Z. Phys. B (in press). 